\title{
Are Metabolites From the Gut Microbiota Capable of Regulating Epigenetic Mechanisms in the Human Parasite Entamoeba histolytica?
}

\author{
Lotem Sarid and Serge Ankri* \\ Department of Molecular Microbiology, Ruth and Bruce Rappaport Faculty of Medicine, Technion, Haifa, Israel
}

The unicellular parasite Entamoeba histolytica inhabits the human gut. It has to adapt to a complex environment that consists of the host microbiota, nutritional stress, oxidative stress, and nitrosative stress. Adaptation to this complex environment is vital for the survival of this parasite. Studies have shown that the host microbiota shapes virulence and stress adaptation in E. histo/ytica. Increasing evidence suggests that metabolites from the microbiota mediate communication between the parasite and microbiota. In this review,

OPEN ACCESS

Edited by:

Souhila Medjkane,

Université de Paris, France

Reviewed by:

Sudipto Saha,

Bose Institute, India

Cristina E Requena,

Andalusian Autonomous Government

of Genomics and Oncological

Research (GENYO), Spain

*Correspondence:

Serge Ankri

sankri@technion.ac.il

Specialty section:

This article was submitted to

Epigenomics and Epigenetics,

a section of the journal

Frontiers in Cell and Developmental

Biology

Received: 22 December 2021

Accepted: 25 January 2022

Published: 01 March 2022

Citation:

Sarid L and Ankri S (2022) Are Metabolites From the Gut Microbiota

Capable of Regulating Epigenetic

Mechanisms in the Human Parasite

Entamoeba histolytica?

Front. Cell Dev. Biol. 10:841586.

doi: 10.3389/fcell.2022.841586 we discuss the bacterial metabolites that regulate epigenetic processes in E. histolytica and the implications that this knowledge may have for the development of new anti-amebic strategies.

Keywords: Entamoeba histolitytica, gut mcirobiota, metabolites, epitranscriptome/epigenome, parasite

\section{INTRODUCTION}

Entamoeba histolytica is a parasitic unicellular organism that causes amebiasis, a disease of the intestinal tract. African and Central American countries with poor sanitation have higher rates of infection. In 2010 it was evaluated that E. histolytica causes 55,500 deaths and 2.237 million disability-adjusted life years (Turkeltaub et al., 2015). Food or water that contains E. histolytica's cysts (the infective form) is the source of the infection. Cysts undergo excystation in the small intestine and trophozoites (the invasive form) are released. These trophozoites will then migrate to the large intestine, where they can colonize, or undergo encystation and be released in the feces. A majority of infections are asymptomatic, but for unknown reasons, the trophozoites can become highly virulent and can cause invasive disease (10\%) (Chou and Austin, 2021). The development of vaccines targeting important E. histolytica antigens, such as Gal-lectin, the serine-rich protein and the $29 \mathrm{kDa}$ reductase antigen, has resulted in partial protection against $E$. histolytica infection in animal models (Quach et al., 2014). There is currently no vaccine for amebiasis. Among the treatment options for amebiasis, metronidazole is the first line of defense, but some side effects, such as diarrhea and anorexia, have been reported (Quach et al., 2014) (Leitsch, 2019). In the lab, metronidazole-resistant strains of E. histolytica have been developed, suggesting that such strains could emerge in the field as well (Wassmann et al., 1999). A population of $10^{14}$ microorganisms inhabits the large intestine. The gut bacteria is associated with unique combinations that are influenced by the physiological conditions within the intestine; most of these are commensal (Lynch and Pedersen, 2016). Trophozoites feed on gut bacteria, and the bacteria are able to influence the virulence of $E$. histolytica (for a recent review see Ankri, 2021). As an example, cultivating E. histolytica with E. coli $\mathrm{O} 55$ can boost $E$. histolytica's virulence which depends on contact between the amoeba and bacteria (Bracha and Mirelman, 1984). Moreover, E. histolytica trophozoites were more resistance to 
oxidative stress (OS) after being incubated with E. coli O55 (Varet et al., 2018). Infection with E. histolytica can cause dysbiosis characterized by a decrease in Lactobacillus and Bacteroides and an increase in Bifidobacterium (Verma et al., 2012). E. histolytica must be capable of adapting to the changing environment in order to survive. Epigenetics deals with the study of changes in gene expression without altering DNA sequence. Epigenetic variation is able to respond to environmental shocks faster than genetic variation derived from evolutionary change (Prentis et al., 2008). A growing body of evidence supports the role of the gut microbiota in the development of pathological conditions, including cancer, through metabolites produced by the microbiota (Zhang et al., 2021). These metabolites have also a direct effect on the innate immune response of the host against $E$. histolytica. For example, deoxycholic acid produced by Clostridium scindens protects mice from E. histolytica infection by affecting granulocyte-monocyte progenitors levels in the bone marrow through epigenetic regulation of the level of intestinal neutrophils (Burgess et al., 2020). In contrast, the impact of microbiota metabolites on parasites is poorly understood. Our review discusses some gut microbiota metabolites and their role in the regulation of epigenetic events in E. histolytica.

\section{CURRENT KNOWLEDGE OF THE EPIGENETIC MECHANISMS CHARACTERIZED IN ENTAMOEBA PARASITES}

\section{Histone Modifications}

An earlier review of the chromatin organization, histone modifications, and their roles in Entamoeba virulence and differentiation has been reported (Tovy and Ankri, 2010). As a result of difficulties in getting pure histones from Entamoeba parasites, development on post-translational modifications in these histones is relatively modest (Lozano-Amado et al., 2016).

\section{Histone Methylation}

The methylation of histone tails occurs on arginine and lysine residues. Lysine methylation in E. histolytica histones regulates epigenetic regulation in this parasite. Inactivation of genes expression was linked to the demethylation of lysine 4 of histone $\mathrm{H} 3$ and the dimethylation of lysine 27 of histone $\mathrm{H} 3$ (Anbar et al., 2005) (Foda and Singh, 2015). Four expressed and catalytically active E. histolytica histone lysine methyltransferases (EhHKMTases) have been characterized (Borbolla-Vazquez et al., 2016). Two of these EhHKMTases, EhHKMT2 and EhHKMT4, may in addition to catalyze epigenetic marks, methylate proteins involved in phagocytosis. EhPRMT1 is an enzyme that catalyzes dimethylation of arginine 3 of histone $\mathrm{H} 4$ in E. histolytica (Borbolla-Vazquez et al., 2015). The importance of this epigenetic mark on the biology of E. histolytica is still not understood. The activity of histone demethylase in E.histolytica has not been adequately studied to date. Arginine residues can be demethylated by peptidyl arginine deiminase 4, which reverses methylation by converting arginine into citrulline
(Cuthbert et al., 2004). A BLAST search of the genome of E.histolytica, however, found no homolog for this enzyme. However, an analysis of the E. histolytica genome reveals that there are three genes that encode SWIRM-domain proteins. SWIRM domains are found in lysine-specific demethylase 1, a protein that helps remove methyl groups from lysine 4 of histone 3 H3K4 (Forneris et al., 2006). Another large group of histone demethylases are the Jumonji C (JmJC) domain-containing enzymes (Cloos et al., 2008). To remove methyl groups, these enzymes undergo hydroxylation through the use of $\mathrm{Fe} 2+$ and oxygen. Interestingly, a search of the AmoebaDB database for Jumonji domain containing proteins revealed 28 candidates in Acanthamoeba and Naegleria parasites, but none in Entamoeba parasites. The oxygen-dependent mechanism of methyl group removal may not be compatible with the physiology of this anaerobic parasite.

\section{Histone Acetylation}

Detection of histone acetylation in Entamoeba parasites has largely been conducted by immunodetection (Ramakrishnan et al., 2004; Isakov et al., 2008), including more recent evidence of acetylation of lysines 5, 8, 12 and 16 in histone H4 of E.histolytica (Lozano-Amado et al., 2016). Histone acetylation and deacetylation are regulated by enzymes called histone acetylases (HATs) and histone deacetylases (HDACs). HATS are classified by their subcellular location within the nucleus and cytoplasm (Lee and Workman, 2007), as well as by their sequence and structure into several distinct families. GNAT (Krtenic et al., 2020) and MYST (Thomas and Voss, 2007) are the most recognized of these families. HDACs fall into four classes: HDACs (class I and II), NAD + -dependent deacetylases SIR2 (class III), and HDAC11 (class IV) (Park and Kim, 2020). The amoeba genome has so far been found to have a GNAT and MYST HAT, as well as a class I HDAC, although it is possible that other unidentified HATs and HDACs may be present as well (Ramakrishnan et al., 2004). Entamoeba invadens, a parasitic reptile used as a model system for studying development, has two class I HDACs that are homologous to human HDAC3. Among these HDAC3s, HDAC3-2 has the potential to play a role in the encystation by regulating gene expression involved in cyst wall formation (Lozano-Amado et al., 2020).

Histone acetylation is involved in Entamoeba parasite physiology as demonstrated by studies using trichostatin A (TSA), a powerful inhibitor of HDAC class I and II (Yoshida et al., 1990). TSA causes hyperacetylation of histones $\mathrm{H} 4$, inhibits encystation of E. invadens and downregulated the expression of enzymes involved in the formation of the cyst wall which suggest that encystation is control by the level of histone $\mathrm{H} 4$ acetylation (Lozano-Amado et al., 2020). On the other hand, TSA induces the expression of cyst wall-synthesis proteins like chitin synthase or the encystationspecific glycoprotein Jacob (Ehrenkaufer et al., 2007). A different response to TSA between E.histolytica and E.invadens could be related to each organism's adaptation to living in its host.

\section{DNA Methylation}

Mammals and other vertebrates methylate DNA in the C5 position of cytosine (m5C) mainly within $\mathrm{CpG}$ dinucleotides. 
TABLE 1 |A bioinformatic search reveals a variety of tRNA modification proteins in E. histolytica (Partial list).

\begin{tabular}{lc} 
Protein name & $\begin{array}{c}\text { Accession } \\
\text { number }\end{array}$ \\
\hline tRNA dihydrouridine synthase 1 & XP_655221 \\
tRNA dihydrouridine synthase 1 & XP_655192 \\
tRNA dihydrouridine synthase 3-like & XP_651736 \\
RNA: NAD 2' phosphotransferase (tRNA splicing) & XP_650634 \\
RNA: NAD 2' phosphotransferase (tRNA splicing) & XP_648371 \\
tRNA nucleotidyltransferase & XP_654783 \\
tRNA nucleotidyltransferase & XP_652076 \\
tRNA delta(2)-isopentenylpyrophosphate transferase & XP_655777 \\
tRNA adenosine deaminase subunit & XP_649973 \\
DNA/tRNA C-5 methyl transferase (Ehmeth) & XP_655267 \\
tRNA (uracil-5) methyl transferase & XP_650594 \\
tRNA (uracil-5) methyl transferase & XP_650606 \\
tRNA (1-methyladenosine) methyltransferase (poss) & XP_653764 \\
tRNA (1-methyladenosine) methyltransferase (poss) & XP_650623 \\
tRNA cytosine methyltransferase (EHI_103830)Trm4/ & XP_653353 \\
EhNSUN2 & \\
tRNA cytosine methyltransferase (EHI_098500) & XP_656026 \\
tRNA/rRNA C-5-methyltransferase (EH__140970) & XP_654608 \\
N2N2-dimethylguanosine tRNA methyltransferase & XP_657464 \\
tRNA methyltransferase subunit TRM5 & XP_650095 \\
tRNA methyltransferase subunit TRM8 & XP_656291 \\
tRNA methyltransferase subunit TRM8 & XP_649121 \\
tRNA methyltransferase subunit TRM10 & XP650920 \\
queuine tRNA-ribosyltransferase & XP_656142 \\
queuine tRNA-ribosyltransferase & XP_652881 \\
D-Tyr-tRNA (Tyr) deacylase (poss) & XP_656041
\end{tabular}

Candidate tRNA:m5C MTases are highlighted in bold font.

The enzymes that catalyzed the formation of this modification are methyl 5-cytosine DNA methyltransferase (Dnmts) and S-adenosylmethionine is their cofactor. DNA methylation pattern maintenance is carried out by Dnmt1, while new DNA methylation occurs by Dnmt3a and B (Lyko, 2018).

The Dnmt2 protein is a member of a large family of proteins found in almost all species. Their activity as tRNA methyltransferases was first described in 2006 (Goll et al., 2006), and has been well recognized ever since (Jeltsch et al., 2017). Although Dnmt2 enzymes methylate tRNA molecules, they use a catalytic mechanism that is similar to that of DNA methyltransferases (Jurkowski et al., 2008). There is a lot of controversy regarding their ability to methylate DNA. The contradictory reports regarding the fruit fly Drosophila melanogaster illustrate this controversy (Phalke et al., 2009; Schaefer and Lyko, 2010; Dunwell and Pfeifer, 2014; Deshmukh et al., 2018). Experimental evidence supports Dnmt2 as a DNA MT enzyme in some parasites including E.histolytica, Schistosoma mansoni and more recently Plasmodium falciparum (Fisher et al., 2004; Geyer et al., 2011; Hammam et al., 2021). Dnmt2-mediated DNA methylation is likely to be involved in the control of repetitive DNA element which are considered genomic parasites (Harony et al., 2006; Lavi et al., 2008; Geyer et al., 2011).

\section{tRNA Methylation}

Epitranscriptomics is field that deals with RNA modification. More than 150 post-synthetic RNA modifications are known to date, addressing all RNA species, which are catalyzed by more than 50 different RNA modifying enzymes (Boccaletto et al., 2018; Frye et al., 2018). tRNA modifications were found to take part in cell biology processes such as in tRNA stability, cellular stress response, drugs resistance and cell growth (Emilsson et al., 1992; Alexandrov et al., 2006; Begley et al., 2007; Netzer et al., 2009; Thompson and Parker, 2009; Motorin and Helm, 2010; Schaefer et al., 2010; Schimmel, 2018; Masuda et al., 2019). E. histolytica genome encodes for many tRNA modifying enzymes including 13 methyl transferases that belong to the NSUN and Dnmt2 family (Table 1). In mammalian, NSUN family consists of seven members, designated as NSUN1-7. Among these seven NSUN members, NSUN1, NSUN4 and NSUN5 could catalyze m5C on rRNA (Metodiev et al., 2014; Bourgeois et al., 2015); NSUN2, NSUN3 and NSUN6 are tRNA:m5C MTases (Brzezicha et al., 2006; Haag et al., 2015; Van Haute et al., 2016). Meanwhile, NSUN2 could also catalyze m5C on mRNA, vault RNA, microRNA and mitochondrial tRNA (Hussain et al., 2013; Yang et al., 2017; Shinoda et al., 2019). NSUN7 could catalyze m5C on enhancer RNA (Aguilo et al., 2016). The biological functions of some mammalian NSUN members have been investigated, revealing roles in protein biosynthesis, cell proliferation and differentiation, and organ development (Chi and Delgado-Olguin, 2013). Concomitantly, aberrant expression of several NSUN members are closely related to diseases (Bohnsack et al., 2019; Chellamuthu and Gray, 2020). In E. histolytica, nothing is known about these modifications or any of these enzymes and their roles during stress response in the parasite except for 5-methylcytosine (m5C) at position 38 of tRNA $^{\text {Asp }}$ (GUC) which is catalyzed by Ehmeth, a Dnmt2-type MTase (Tovy et al., 2010). A recent review about Dnmt2 shows that the effect of this enzyme on tRNA methylation are multiple (Jeltsch et al., 2017) and it includes an effect on protein translation (Tuorto et al., 2012) and on the production of specific stress-induced tRNA-derived small RNAs. E. histolytica is capable of responding to changes in its surrounding glucose concentration: short term glucose starvation $(12 \mathrm{~h})$ led to the accumulation of enolase, a glycolytic enzyme, in the nucleus. Enolase interacted with the catalytic site of Ehmeth, subsequently inhibiting its tRNA MTase activity (Tovy et al., 2010). Overexpression of Ehmeth confers resistance to oxidative stress (OS) (Fisher et al., 2006) and nitrosative stress (NS) (Hertz et al., 2014). Ehmethmediated resistance to NS is associated with 1) high levels of tRNA $^{\text {Asp }}$ (GUC) methylation, 2) persistence of protein synthesis under conditions of NS, and 3) the specific expression of proteins which are involved in protein translation, protein transport, vacuolar sorting signaling, and resistance to OS and NS, such as alcohol dehydrogenase 2 (ADH2) and peroxyredoxin (Hertz et al., 2014).

\section{RNA-Mediated Silencing}

In order to achieve RNA-mediated silencing in E.histolytica, several methods are currently used, including double-stranded RNA (Solis et al., 2009), short-hairpin RNA (Linford et al., 2009), trans-inactivation (G3 strain) (Bujanover et al., 2003), and trigger-induced RNAi (Morf et al., 2013).

DICER and RISC complex are used in the first two methods (Paturi and Deshmukh, 2021). Despite lacking a gene matching 
the canonical Dicer structure, E. histolytica does express an RnaseIII protein (EHI_068740) (Abed and Ankri, 2005; Pompey et al., 2015). Of the three AGO genes, only one (EHI_125650) is highly expressed in the parasite (Zhang et al., 2011). Small RNAs of $27 \mathrm{nt}$ are associated with the AGO protein, providing evidence for a RNA-mediated silencing system in $E$. histolytica (Zhang et al., 2011).

G3 strain based gene silencing uses an E.histolytica strain that has been transfected with an upstream region of Ehap-a in order to silence expression of the amoebapore A gene (Bujanover et al., 2003). Even after removing the selectable marker, the silencing is stable, and the plasmidless gene-silenced clone G3 can be used to silence another gene. The presence of demethylated K4 in histone $\mathrm{H} 3$, a marker for inactive euchromatic regions, near the Ehap-a gene is consistent with transcriptional inactivation of that gene (Mirelman et al., 2006)

Small RNAs are used in trigger-induced RNAi to silence specific genes by sequence-specific silencing. Since the levels of antisense small RNAs correlate inversely with the mRNA expression levels of their cognate target genes, these small RNAs are thought to play an important role in gene silencing (Matthiesen et al., 2019). Demethylation of K27 in histone H3, a repressive histone mark, is enriched at genes silenced by RNAi (Foda and Singh, 2015).

\section{Possible Roles of Microbiota Metabolites in Shaping the Epigenetic Mechanisms in E.histolytica \\ Folate and S-Adenosylmethionine}

Folate (or vitamin B9) is a central agent in the formation of SAM (Bailey and Gregory, 1999; Abbasi et al., 2018) by providing the methyl groups following the re-methylation of homocysteine to methionine (Kim, 2005; Krautkramer et al., 2017). SAM is a crucial cofactor participating in enzyme catalysis of many methyltransferases (MTases). In the host, SAM originates from methionine, an essential amino acid obtained through nutrition (Poirier et al., 2001). Folate is in part produced by the gut microbiota (Engevik et al., 2019). It has been suggested that dysbiosis of the gut microbiota can affect SAM levels and, therefore, modify MTases activity. Lactic acid bacteria naturally present in the human gut or probiotics are recognized for their ability to produce folate (Pompei et al., 2007). These bacteria may have an impact in the overall level of folate in the host and consequently on the level of SAM. E. histolytica requires folate for growth in axenic conditions (Diamond et al., 1978), possibly indicating the parasite is dependent upon the gut microbiota for folate production. L-cysteine pool in the gut depends on dietary habits (Bauchart-Thevret et al., 2009), uptake by the host (Banjac et al., 2008), de novo synthesis or degradation by the gut microbiota into hydrogen sulfide (Neis et al., 2015; Braccia et al., 2021). Deprivation L-cysteine in E. histolytica results in a significant decrease in SAM levels (Husain et al., 2010) which may affect the activity of MTases. These data strongly suggest that the gut microbiota influences the level of SAM in E. histolytica and, as a result, the activity of its MTases.
It is still not completely understood how tRNA ${ }^{\text {Asp }}$ (GUC) methylation regulates the synthesis of these proteins. However, this regulation might depend on the SAM level dictated by the gut microbiota. An example to support this notion has been provided by changes in DNA methylation observed in cocultivated human fetal and adult intestinal epithelial cells exposed to folate producing bacteria Lactobacillus acidophilus and Bifidobacterium infantis (Cortese et al., 2016; Qin and Wade, 2018). Recently, a new mechanism that links tRNA methylation to the gut microbiota activity has been revealed. This mechanism relies on queuine, a micronutriement produced by the gut microbiota.

\section{Queuine}

Queuosine (Q) and its glycosylated derivatives occur in position 34 of the anticodon of tRNA ${ }^{\text {Asp }}, \mathrm{tRNA}^{\mathrm{His}} \mathrm{tRNA}^{\mathrm{Asn}}$ and $\mathrm{tRNA}^{\mathrm{Tyr}}$ of eubacteria and eukaryotes except for Saccharomyces cerevisiae (Walden et al., 1982; Fergus et al., 2015). Q is highly conserved and found in plants, fishes, insects and mammals. While many bacteria can synthesize queuine (the nucleobase of $\mathrm{Q}$ ) de novo, salvage of the prokaryotic $Q$ precursors $\mathrm{preQ}_{0}$ and $\mathrm{preQ}_{1}$ has recently be reported (Yuan et al., 2019). Eukaryotes are not capable of $\mathrm{Q}$ synthesis and rely on salvage of the queuine base as a Q precursor either by nutrition or by the intestinal bacterial flora (Farkas, 1980; Katze et al., 1982; Ott et al., 1982). Queuine has been associated with neuroprotection (Richard et al., 2021) whereas absence of queuine is associated with better cancer cells growth and survival by promoting Warburg metabolism (Hayes et al., 2020). The tRNA-guanine transglycosylase (TGT) is the main enzyme responsible for the formation of $\mathrm{Q}$ in the anticodon loop position 34 of $\mathrm{tRNA}^{\mathrm{Asp}}$, $\mathrm{tRNA}^{\mathrm{His}}$, $\mathrm{tRNA}^{\mathrm{Asn}}$ and $\mathrm{tRNA}^{\mathrm{Tyr}}$. The enzyme exchanges G34 for the precursors. The cyclopentendiol moiety is synthesized at the level of tRNA from unknown precursors and enzymes in both eubacterial and eukaryotic species. The crystal structure of TGT from Zymomonas mobilis comprises an irregular $(\beta / \alpha)_{8}$ TIM barrel with a C-terminal zinc binding subdomain (Stengl et al., 2007). In contrast to eubacterial TGT enzymes, all of which are homodimers, eukaryotic TGT enzymes, such as human TGT, are heterodimers and consist of a Q tRNA-ribosyltransferase 1 (QTRT1, eubacterial TGT homolog) and a Q tRNA-ribosyltransferase domain-containing 1 (QTRTD1) (Stengl et al., 2007; Chen et al., 2010). Both subunits are homologous to the bacterial domain QTRT; however, while QTRT1 should be catalytically active, the QTRTD1 subunit has lost residues important both for binding and catalysis. The source of transglycosylase activity of human TGT is the $h$ QTRT1-hQTRTD1 heterodimer, while $h$ QTRT1 and hQTRTD1 monomers do not exhibit any activity (Chen et al., 2010). Both $h$ QTRT1 and $h$ QTRTD1 co-localize in mitochondria (Boland et al., 2009). The crystal structure of hQTRTD1 (also called QTRT2) revealed that $h$ QTRTD1 forms a homodimer with striking similarity to that of bacterial TGT (Behrens et al., 2018). Recently, the crystal structure of hTGT in its heterodimeric form and in complex with a 25-mer stem loop RNA has been established (Sievers et al., 2021). The detailed analysis of its dimer interface and interaction with a minimal substrate RNA indicates that one base only, guanine 34 or queuine, can simultaneously reside at the active site in support to a "ping-pong" mechanism that has already been 


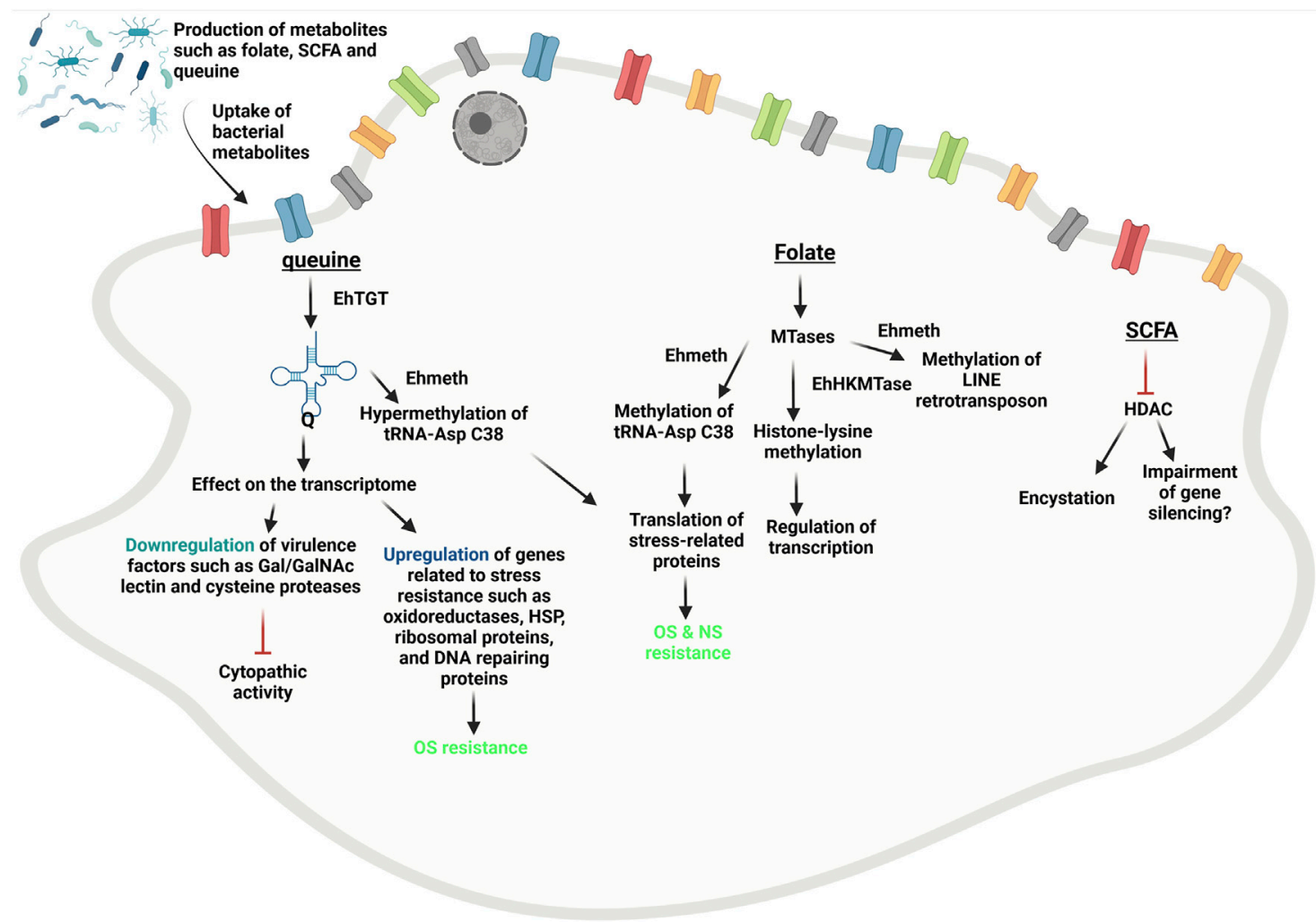

FIGURE 1 | Legend summary of the effect of bacterial-metabolites on E. histolytica epigenome. Queuine incorporated into specific tRNA by EhTGT and lead to change in the transcriptome, OS resistance, hypermethylation of tRNAAspC38 and impaired virulence of E. histolytica trophozoites (Nagaraja et al., 2021). SCFA are HDAC inhibitors (Waldecker et al., 2008). SCFA promote encystation of $E$. histolytica (Wesel et al., 2021). Folate is critical for the formation of SAM which is used as cofactor of MTases (Abbasi et al., 2018). Lysine methylation in histones is catalyzed by EhHKMTases and demethylation of H3-lysine 4 is often indicative of inactivation of genes expression (Anbar et al., 2005). Ehmeth is a Dnmt2 homolog that can methylate LINE retrotransposon (Fisher et al., 2004; Harony et al., 2006) and tRNAAspC38 which is the favorite Ehmeth substrate (Tovy et al., 2010). Ehmeth activity is correlated to OS\&NS resistance probably through the regulation of stressrelated proteins translation (Fisher et al., 2006; Hertz et al., 2014). This document has be created with BioRender.com (accessed on December 21, 2021).

proposed for E.coli TGT (Goodenough-Lashua and Garcia, 2003). Regarding hQTRTD1, the authors proposed that it could serve to anchor the TGT enzyme in the compartmentalized eukaryotic cell (Sievers et al., 2021). Based on the annotation of the E. histolytica genome, a homolog of hQTRT1 and hQTRTD1 exists in $E$. histolytica, namely EhQTRT1 (XP_656142.1) and EhQTRTD1 (XP_652881.1). E.histolytica tRNA-guanine transglycosylase (TGT) is a heterodimer composed of EhQTRT1 and EhQTRTD1 (Nagaraja et al., 2021). EhTGT is catalytically active and it incorporates queuine into E. histolytica tRNAs. The presence of $\mathrm{Q}$ in tRNA $\mathrm{AUC}_{\mathrm{GUC}}^{\mathrm{Asp}}$ stimulates its methylation by Ehmeth, a Dnmt2-type multisubstrate tRNA methyltransferase, at the C38 position. Queuine does not affect the growth of the parasite, it protects the parasite against oxidative stress (OS) and it antagonizes the negative effect that OS has on translation by inducing the expression of genes involved in OS response, such as heat shock protein 70 (Hsp 70), antioxidant enzymes, and enzymes involved in DNA repair. On the other hand, queuine impairs E. histolytica virulence determined in mouse model of amebic colitis by downregulating the expression of genes previously associated with virulence, including cysteine proteases, cytoskeletal proteins, and small GTPases. Silencing of EhTGT expression prevents the incorporation of queuine into
tRNAs, impairs the methylation of $\mathrm{C} 38$ in $\mathrm{tRNA}_{\mathrm{GUC}}^{\mathrm{Asp}}$, inhibits the growth of the parasite, impairs its resistance to OS and its cytopathic activity. Information about how Q is salvage by eukaryotic organisms is scanty. In mammalian cells, queuine is generated from Q- $5^{\prime}$-phosphate which suggests that salvage $\mathrm{Q}$ base is coming from tRNA degraded during the normal turnover process (Gunduz and Katze, 1984). In the green algae Chlorella pyrenoidosa and Chlamydomonas reinhardtii an enzymatic activity that catalyzes the cleavage of $\mathrm{Q}$ has been identified but its nature is not known (Kirtland et al., 1988). A search of genes that co-distribute with eukaryotic QTRT1 and QTRTD1 identified a potential Q salvage protein, DUF2419 (Zallot et al., 2014). The structural similarity of DUF2419 with DNA glycosylases suggests a ribonucleoside hydrolase activity. Indeed, genetic evidences support the role of DUF2419 as a Q salvaging enzyme in Schizosaccharomyces pombe, human, maize, and Streptococcus thermophilus (Zallot et al., 2014).

\section{Short Chain Fatty Acids}

Increasing evidences support the role of SCFA which are exclusively produced by the gut microbiota from the 
catabolism of carbohydrates of dietary origin in the regulation of global histone acetylation and methylation in the host (Krautkramer et al., 2016; Kelly et al., 2018). The abundances of SCFA depends on the composition of the microbiota with the dominant-SCFA producing bacteria being Faecalibacterium prausnitzii (Lopez-Siles et al., 2017) and Roseburia intestinalis (La Rosa et al., 2019). Acetate and propionate are important SCFA that inhibit histone deacetylases (HDACs) (Chen et al., 2003; Maslowski and Mackay, 2011) leading to histone decondensation, chromatin relaxation and active transcriptional state (Chen et al., 2003). Butyrate, another SCFA, inhibits HDACs by binding to $\mathrm{Zn}^{+2}$ that is located in their active site (Davie, 2003). Gut microbiota dysbiosis is associated with many intestinal and extra-intestinal disorders including bowel disease, metabolic syndrome, obesity, cardiovascular disease (Carding et al., 2015) and neurodegenerative disorders (Aho et al., 2021). Many of these disorders may have been triggered by a dysregulation of SCFA production consequent to dysbiosis (Tan et al., 2014). E. histolytica causes dysbiosis in the human gut by feeding on preferred bacteria like Lactobacillus ruminus (Iyer et al., 2019) and SCFA producing bacteria like Bifidobacterium longum (Iyer et al., 2019). Therefore, it is possible that E. histolytica infection change the level of SCFA in the host; however experimental evidences are needed to confirm this hypothesis.

SCFA have a direct effect on E.histolytica by promoting its encystation (Wesel et al., 2021). By consuming SCFA-producing flora, the parasite may control its entry into encystation through a modulation of its HDAC. It is important to note that SCFA have the opposite effect on the encystation of Entamoeba invadens, a reptile parasite (Byers et al., 2005). Entamoeba parasites may have evolved to respond differentially to SCFA produced by the microbiota of their respective host.

As mentioned earlier, repressive histone marks (demethylated K4 and K27 in histone H3) are present in the vicinity of silenced genes in E.histolytica. Consequently, one must speculate that SCFA affects gene silencing by inhibiting histone deacetylase activity. There is, however, a need to be cautious before jumping to conclusions, as reversal of the silencing seems to be resistant to TSA treatment (Bracha et al., 2003).

\section{CONCLUSION AND PERSPECTIVES}

An increasing number of studies support an intricate relationship between the gut microbiota and E. histolytica (Burgess and Petri, 2016; Ankri, 2021) and significant changes in the gut microbiota has been associated with amebiasis (Rani et al., 2006; Verma et al.,

\section{REFERENCES}

Abbasi, I. H. R., Abbasi, F., Wang, L., Abd El Hack, M. E., Swelum, A. A., Hao, R., et al. (2018). Folate Promotes S-Adenosyl Methionine Reactions and the Microbial Methylation Cycle and Boosts Ruminants Production and Reproduction. AMB Expr. 8 (1), 65. doi:10.1186/s13568-018-0592-5
2012). For example, the presence of Prevotella copri, in gut flora was associated with E. histolytica induced diarrheal disease in children (Ngobeni et al., 2017). These characteristics may serve as biomarkers for screening of amebiasis and prognosis. The gut microbiota impacts probably the manifestation and development of amebiasis through immunity (Burgess and Petri, 2016; Watanabe et al., 2017; Burgess et al., 2020), metabolism (Nakada-Tsukui and Nozaki, 2016), direct interaction with the parasite (Bracha and Mirelman, 1984; Padilla-Vaca et al., 1999; Galvan-Moroyoqui et al., 2008; Varet et al., 2018), metabolites that promotes stress resistance in the parasite (Shaulov et al., 2018; Nagaraja et al., 2021) and modulate epigenetic functions (this review). Fine-tuning the gut microbiota through diet or probiotics has been considered as a possible strategy to improve the efficacy and decrease the toxicity of treatment against cancer (for a recent review see (Zhou et al., 2021)). The same strategy has been considered for the prevention of amebiasis (Nagaraja and Ankri, 2019; Ankri, 2021). Some studies proposed to use probiotics to inhibit the binding of the parasite to intestinal mucosa surface (Rigothier et al., 1994; Mansour-Ghanaei et al., 2003) or to benefit from their amebicide activity (Varet et al., 2018). However, the idea of using probiotics and their metabolites to manipulate the epigenome of E.histolytica is just emerging. As summarized in Figure 1, we have discussed a number of metabolites that may be used for this purpose. By impairing the virulence of E. histolytica, queuine is a serious candidate. Yet, many questions remain about the choice of the probiotic queuine donor, the possibility to use queuine as postbiotic and the safety of this approach to human. Regarding safety, probiotics are generally considered harmless for immunocompetent individuals but sepsis in immunocompromised individuals or critically ill patients has been reported (Doron and Snydman, 2015). Finally, we need to be cautious in extrapolating the conclusions drawn about probiotics and their metabolites from animal experimentations to human.

\section{AUTHOR CONTRIBUTIONS}

LS and SA contributed substantially to the conception and design of this review and wrote the manuscript

\section{FUNDING}

This work was supported by the Israel Science Foundation (3208/19) and the Ministry of Science and Technology, Israel (1020546).

Abed, M., and Ankri, S. (2005). Molecular Characterization of Entamoeba Histolytica RNase III and AGO2, Two RNA Interference Hallmark Proteins. Exp. Parasitol. 110 (3), 265-269. doi:10.1016/j.exppara.2005.02.023

Aguilo, F., Li, S., Balasubramaniyan, N., Sancho, A., Benko, S., Zhang, F., et al. (2016). Deposition of 5-Methylcytosine on Enhancer RNAs Enables the Coactivator Function of PGC-1 $\alpha$. Cel. Rep. 14 (3), 479-492. doi:10.1016/j. celrep.2015.12.043 
Aho, V. T. E., Houser, M. C., Pereira, P. A. B., Chang, J., Rudi, K., Paulin, L., et al. (2021). Relationships of Gut Microbiota, Short-Chain Fatty Acids, Inflammation, and the Gut Barrier in Parkinson's Disease. Mol. Neurodegeneration 16 (1), 6. doi:10.1186/s13024-021-00427-6

Alexandrov, A., Chernyakov, I., Gu, W., Hiley, S. L., Hughes, T. R., Grayhack, E. J., et al. (2006). Rapid tRNA Decay Can Result from Lack of Nonessential Modifications. Mol. Cel. 21 (1), 87-96. doi:10.1016/j.molcel.2005.10.036

Anbar, M., Bracha, R., Nuchamowitz, Y., Li, Y., Florentin, A., and Mirelman, D. (2005). Involvement of a Short Interspersed Element in Epigenetic Transcriptional Silencing of the Amoebapore Gene in Entamoeba Histolytica. Eukaryot. Cel. 4 (11), 1775-1784. doi:10.1128/ec.4.11.1775-1784. 2005

Ankri, S. (2021). Entamoeba Histolytica-Gut Microbiota Interaction: More Than Meets the Eye. Microorganisms 9 (3), 581. doi:10.3390/microorganisms 9030581

Bailey, L. B., and Gregory, J. F., 3rd (1999). Folate Metabolism and Requirements. J. Nutr. 129 (4), 779-782. doi:10.1093/jn/129.4.779

Banjac, A., Perisic, T., Sato, H., Seiler, A., Bannai, S., Weiss, N., et al. (2008). The Cystine/cysteine Cycle: a Redox Cycle Regulating Susceptibility versus Resistance to Cell Death. Oncogene 27 (11), 1618-1628. doi:10.1038/sj.onc. 1210796

Bauchart-Thevret, C., Stoll, B., and Burrin, D. G. (2009). Intestinal Metabolism of Sulfur Amino Acids. Nutr. Res. Rev. 22 (2), 175-187. doi:10.1017/ S0954422409990138

Begley, U., Dyavaiah, M., Patil, A., Rooney, J. P., DiRenzo, D., Young, C. M., et al. (2007). Trm9-catalyzed tRNA Modifications Link Translation to the DNA Damage Response. Mol. Cel. 28 (5), 860-870. doi:10.1016/j.molcel.2007.09.021

Behrens, C., Biela, I., Petiot-Bécard, S., Botzanowski, T., Cianférani, S., Sager, C. P., et al. (2018). Homodimer Architecture of QTRT2, the Noncatalytic Subunit of the Eukaryotic tRNA-Guanine Transglycosylase. Biochemistry 57, 3953-3965. doi:10.1021/acs.biochem.8b00294

Boccaletto, P., Machnicka, M. A., Purta, E., Piątkowski, P., Bagiński, B., Wirecki, T. K., et al. (2018). MODOMICS: a Database of RNA Modification Pathways. 2017 Update. Nucleic Acids Res. 46 (D1), D303-D307. doi:10.1093/nar/gkx1030

Bohnsack, K., Höbartner, C., and Bohnsack, M. (2019). Eukaryotic 5methylcytosine (m5C) RNA Methyltransferases: Mechanisms, Cellular Functions, and Links to Disease. Genes 10 (2), 102. doi:10.3390/genes10020102

Boland, C., Hayes, P., Santa-Maria, I., Nishimura, S., and Kelly, V. P. (2009). Queuosine Formation in Eukaryotic tRNA Occurs via a MitochondriaLocalized Heteromeric Transglycosylase. J. Biol. Chem. 284 (27), 18218-18227. doi:10.1074/jbc.M109.002477M109.002477

Borbolla-Vázquez, J., Orozco, E., Betanzos, A., and Rodríguez, M. A. (2015). Entamoeba Histolytica: Protein Arginine Transferase 1a Methylates Arginine Residues and Potentially Modify the H4 Histone. Parasites Vectors 8, 219. doi:10.1186/s13071-015-0820-7

Borbolla-Vázquez, J., Orozco, E., Medina-Gómez, C., Martínez-Higuera, A., JavierReyna, R., Chávez, B., et al. (2016). Identification and Functional Characterization of Lysine Methyltransferases ofEntamoeba Histolytica. Mol. Microbiol. 101 (2), 351-365. doi:10.1111/mmi.13394

Bourgeois, G., Ney, M., Gaspar, I., Aigueperse, C., Schaefer, M., Kellner, S., et al. (2015). Eukaryotic rRNA Modification by Yeast 5-MethylcytosineMethyltransferases and Human Proliferation-Associated Antigen P120. PLoS One 10 (7), e0133321. doi:10.1371/journal.pone.0133321

Braccia, D. J., Jiang, X., Pop, M., and Hall, A. B. (2021). The Capacity to Produce Hydrogen Sulfide (H2S) via Cysteine Degradation Is Ubiquitous in the Human Gut Microbiome. Front. Microbiol. 12, 705583. doi:10.3389/ fmicb.2021.705583

Bracha, R., and Mirelman, D. (1984). Virulence of Entamoeba Histolytica Trophozoites. Effects of Bacteria, Microaerobic Conditions, and Metronidazole. J. Exp. Med. 160 (2), 353-368. doi:10.1084/jem.160.2.353

Bracha, R., Nuchamowitz, Y., and Mirelman, D. (2003). Transcriptional Silencing of an Amoebapore Gene in Entamoeba Histolytica : Molecular Analysis and Effect on Pathogenicity. Eukaryot. Cel 2 (2), 295-305. doi:10.1128/ec.2.2.295-305.2003

Brzezicha, B., Schmidt, M., Makałowska, I., Jarmołowski, A., Pieńkowska, J., and Szweykowska-Kulińska, Z. (2006). Identification of Human tRNA:m5C Methyltransferase Catalysing Intron-dependent m5C Formation in the First Position of the Anticodon of the $\$\{\text { lhbox }\{\text { Pre-tRNA }\}\}_{-}\{$\{left( $($hbox $\{$CAA \} $\mid$ right $)\}\{$ hboox $\{$ Leu $\}\} \$$. Nucleic Acids Res. 34 (20), 6034-6043. doi:10.1093/ nar/gkl765
Bujanover, S., Katz, U., Bracha, R., and Mirelman, D. (2003). A Virulence Attenuated Amoebapore-Less Mutant of Entamoeba Histolytica and its Interaction with Host Cells. Int. J. Parasitol. 33 (14), 1655-1663. doi:10. 1016/s0020-7519(03)00268-6

Burgess, S. L., Leslie, J. L., Uddin, J., Oakland, D. N., Gilchrist, C., Moreau, G. B., et al. (2020). Gut Microbiome Communication with Bone Marrow Regulates Susceptibility to Amebiasis. J. Clin. Invest. 130 (8), 4019-4024. doi:10.1172/ JCI133605

Burgess, S. L., and Petri, W. A., Jr. (2016). The Intestinal Bacterial Microbiome and E. Histolytica Infection. Curr. Trop. Med. Rep. 3, 71-74. doi:10.1007/s40475016-0083-1

Byers, J., Faigle, W., and Eichinger, D. (2005). Colonic Short-Chain Fatty Acids Inhibit Encystation of Entamoeba Invadens. Cel. Microbiol. 7 (2), 269-279. doi:10.1111/j.1462-5822.2004.00457.x

Carding, S., Verbeke, K., Vipond, D. T., Corfe, B. M., and Owen, L. J. (2015). Dysbiosis of the Gut Microbiota in Disease. Microb. Ecol. Health Dis. 26, 26191. doi:10.3402/mehd.v26.26191

Chellamuthu, A., and Gray, S. G. (2020). The RNA Methyltransferase NSUN2 and its Potential Roles in Cancer. Cells 9 (8), 1758. doi:10.3390/cells9081758

Chen, J., Faller, D., and Spanjaard, R. (2003). Short-chain Fatty Acid Inhibitors of Histone Deacetylases: Promising Anticancer Therapeutics?. Ccdt 3 (3), 219-236. doi:10.2174/1568009033481994

Chen, Y.-C., Kelly, V. P., Stachura, S. V., and Garcia, G. A. (2010). Characterization of the Human tRNA-Guanine Transglycosylase: Confirmation of the Heterodimeric Subunit Structure. RNA 16 (5), 958-968. doi:10.1261/rna. 1997610rna.1997610

Chi, L., and Delgado-Olguín, P. (2013). Expression of NOL1/NOP2/sun Domain (Nsun) RNA Methyltransferase Family Genes in Early Mouse Embryogenesis. Gene Expr. Patterns 13 (8), 319-327. doi:10.1016/j.gep.2013.06.003

Chou, A., and Austin, R. L. (2021). "Entamoeba Histolytica," in StatPearls (Treasure Island (FL).

Cloos, P. A. C., Christensen, J., Agger, K., and Helin, K. (2008). Erasing the Methyl Mark: Histone Demethylases at the center of Cellular Differentiation and Disease. Genes Dev. 22 (9), 1115-1140. doi:10.1101/gad.1652908

Cortese, R., Lu, L., Yu, Y., Ruden, D., and Claud, E. C. (2016). EpigenomeMicrobiome Crosstalk: A Potential New Paradigm Influencing Neonatal Susceptibility to Disease. Epigenetics 11 (3), 205-215. doi:10.1080/15592294. 2016.1155011

Cuthbert, G. L., Daujat, S., Snowden, A. W., Erdjument-Bromage, H., Hagiwara, T., Yamada, M., et al. (2004). Histone Deimination Antagonizes Arginine Methylation. Cell 118 (5), 545-553. doi:10.1016/j.cell.2004.08.020

Davie, J. R. (2003). Inhibition of Histone Deacetylase Activity by Butyrate. J. Nutr. 133 (7 Suppl. 1), 2485S-2493S. doi:10.1093/jn/133.7.2485S

Deshmukh, S., Ponnaluri, V. C., Dai, N., Pradhan, S., and Deobagkar, D. (2018). Levels of DNA Cytosine Methylation in theDrosophilagenome. PeerJ 6, e5119. doi: $10.7717 /$ peeri.5119

Diamond, L. S., Harlow, D. R., and Cunnick, C. C. (1978). A New Medium for the Axenic Cultivation of Entamoeba Histolytica and Other Entamoeba. Trans. $R$. Soc. Trop. Med. Hyg. 72 (4), 431-432. doi:10.1016/0035-9203(78)90144-x

Doron, S., and Snydman, D. R. (2015). Risk and Safety of Probiotics. Clin. Infect. Dis. 60 (Suppl. 2), S129-S134. doi:10.1093/cid/civ085

Dunwell, T. L., and Pfeifer, G. P. (2014). Drosophila Genomic Methylation: New Evidence and New Questions. Epigenomics 6 (5), 459-461. doi:10.2217/epi.14.46

Ehrenkaufer, G. M., Eichinger, D. J., and Singh, U. (2007). Trichostatin A Effects on Gene Expression in the Protozoan Parasite Entamoeba Histolytica. BMC Genomics 8, 216. doi:10.1186/1471-2164-8-216

Emilsson, V., Näslund, A. K., and Kurlad, C. G. (1992). Thiolation of Transfer RNA inEscherichia Colivaries with Growth Rate. Nucl. Acids Res. 20 (17), 4499-4505. doi:10.1093/nar/20.17.4499

Engevik, M. A., Morra, C. N., Röth, D., Engevik, K., Spinler, J. K., Devaraj, S., et al. (2019). Microbial Metabolic Capacity for Intestinal Folate Production and Modulation of Host Folate Receptors. Front. Microbiol. 10, 2305. doi:10.3389/ fmicb.2019.02305

Farkas, W. R. (1980). Effect of Diet on the Queuosine Family of tRNAs of Germ-free Mice. J. Biol. Chem. 255 (14), 6832-6835. doi:10.1016/s0021-9258(18)43648-4

Fergus, C., Barnes, D., Alqasem, M., and Kelly, V. (2015). The Queuine Micronutrient: Charting a Course from Microbe to Man. Nutrients 7 (4), 2897-2929. doi:10.3390/nu7042897 
Fisher, O., Siman-Tov, R., and Ankri, S. (2004). Characterization of Cytosine Methylated Regions and 5-cytosine DNA Methyltransferase (Ehmeth) in the Protozoan Parasite Entamoeba Histolytica. Nucleic Acids Res. 32 (1), 287-297. doi:10.1093/nar/gkh16132/1/287

Fisher, O., Siman-Tov, R., and Ankri, S. (2006). Pleiotropic Phenotype in Entamoeba Histolytica Overexpressing DNA Methyltransferase (Ehmeth). Mol. Biochem. Parasitol. 147 (1), 48-54. doi:10.1016/j.molbiopara.2006.01.007

Foda, B. M., and Singh, U. (2015). Dimethylated H3K27 Is a Repressive Epigenetic Histone Mark in the Protist Entamoeba Histolytica and Is Significantly Enriched in Genes Silenced via the RNAi Pathway. J. Biol. Chem. 290 (34), 21114-21130. doi:10.1074/jbc.M115.647263

Forneris, F., Binda, C., Dall'Aglio, A., Fraaije, M. W., Battaglioli, E., and Mattevi, A. (2006). A Highly Specific Mechanism of Histone H3-K4 Recognition by Histone Demethylase LSD1. J. Biol. Chem. 281 (46), 35289-35295. doi:10. 1074/jbc.m607411200

Frye, M., Harada, B. T., Behm, M., and He, C. (2018). RNA Modifications Modulate Gene Expression during Development. Science 361 (6409), 1346-1349. doi:10. 1126/science.aaul646

Galván-Moroyoqui, J. M., Domínguez-Robles, M. d. C., Franco, E., and Meza, I. (2008). The Interplay between Entamoeba and Enteropathogenic Bacteria Modulates Epithelial Cell Damage. Plos Negl. Trop. Dis. 2 (7), e266. doi:10. 1371/journal.pntd.0000266

Geyer, K. K., Rodríguez López, C. M., Chalmers, I. W., Munshi, S. E., Truscott, M., Heald, J., et al. (2011). Cytosine Methylation Regulates Oviposition in the Pathogenic Blood Fluke Schistosoma Mansoni. Nat. Commun. 2, 424. doi:10. 1038/ncomms1433

Goll, M. G., Kirpekar, F., Maggert, K. A., Yoder, J. A., Hsieh, C. L., Zhang, X. Y., et al. (2006). Methylation of tRNA(AsP) by the DNA methyltransferase homolog Dnmt2. Science 311 (5759), 395-398. doi:10.1126/science.1120976

Goodenough-Lashua, D. M., and Garcia, G. A. (2003). tRNA-Guanine Transglycosylase from E. coli: a Ping-Pong Kinetic Mechanism Is Consistent with Nucleophilic Catalysis. Bioorg. Chem. 31 (4), 331-344. doi:10.1016/s00452068(03)00069-5

Gündüz, U., and Katze, J. R. (1984). Queuine Salvage in Mammalian Cells. Evidence that Queuine Is Generated from Queuosine 5'-phosphate. J. Biol. Chem. 259 (2), 1110-1113. doi:10.1016/s0021-9258(17)43573-3

Haag, S., Warda, A. S., Kretschmer, J., Günnigmann, M. A., Höbartner, C., and Bohnsack, M. T. (2015). NSUN6 Is a Human RNA Methyltransferase that Catalyzes Formation of m5C72 in Specific tRNAs. RNA 21 (9), 1532-1543. doi:10.1261/rna.051524.115

Hammam, E., Sinha, A., Baumgarten, S., Nardella, F., Liang, J., Miled, S., et al. (2021). Malaria Parasite Stress Tolerance Is Regulated by DNMT2-Mediated tRNA Cytosine Methylation. mBio 12, e0255821. doi:10.1128/mBio. 02558-21

Harony, H., Bernes, S., Siman-Tov, R., and Ankri, S. (2006). DNA Methylation and Targeting of LINE Retrotransposons in Entamoeba Histolytica and Entamoeba Invadens. Mol. Biochem. Parasitol. 147 (1), 55-63. doi:10.1016/j.molbiopara. 2006.02.005

Hayes, P., Fergus, C., Ghanim, M., Cirzi, C., Burtnyak, L., McGrenaghan, C. J., et al. (2020). Queuine Micronutrient Deficiency Promotes Warburg Metabolism and Reversal of the Mitochondrial ATP Synthase in Hela Cells. Nutrients 12 (3), 871. doi:10.3390/nu12030871

Hertz, R., Tovy, A., Kirschenbaum, M., Geffen, M., Nozaki, T., Adir, N., et al. (2014). The Entamoeba Histolytica Dnmt2 Homolog (Ehmeth) Confers Resistance to Nitrosative Stress. Eukaryot. Cel. 13 (4), 494-503. doi:10.1128/EC.00031-14

Husain, A., Sato, D., Jeelani, G., Mi-ichi, F., Ali, V., Suematsu, M., et al. (2010). Metabolome Analysis Revealed Increase in S-Methylcysteine and Phosphatidylisopropanolamine Synthesis upon L-Cysteine Deprivation in the Anaerobic Protozoan Parasite Entamoeba Histolytica. J. Biol. Chem. 285 (50), 39160-39170. doi:10.1074/jbc.M110.167304M110.167304

Hussain, S., Sajini, A. A., Blanco, S., Dietmann, S., Lombard, P., Sugimoto, Y., et al. (2013). NSun2-mediated Cytosine-5 Methylation of Vault Noncoding RNA Determines its Processing into Regulatory Small RNAs. Cel. Rep. 4 (2), 255-261. doi:10.1016/j.celrep.2013.06.029S2211-1247(13)00321-5

Isakov, E., Siman-Tov, R., Weber, C., Guillen, N., and Ankri, S. (2008). Trichostatin A Regulates Peroxiredoxin Expression and Virulence of the Parasite Entamoeba Histolytica次. Mol. Biochem. Parasitol. 158 (1), 82-94. doi:10.1016/j. molbiopara.2007.11.014S0166-6851(07)00332-5
Iyer, L. R., Verma, A. K., Paul, J., and Bhattacharya, A. (2019). Phagocytosis of Gut Bacteria by Entamoeba Histolytica. Front. Cel. Infect. Microbiol. 9, 34. doi:10. 3389/fcimb.2019.00034

Jeltsch, A., Ehrenhofer-Murray, A., Jurkowski, T. P., Lyko, F., Reuter, G., Ankri, S., et al. (2017). Mechanism and Biological Role of Dnmt2 in Nucleic Acid Methylation. RNA Biol. 14 (9), 1108-1123. doi:10.1080/15476286.2016. 1191737

Jurkowski, T. P., Meusburger, M., Phalke, S., Helm, M., Nellen, W., Reuter, G., et al. (2008). Human DNMT2 Methylates tRNAAsp Molecules Using a DNA Methyltransferase-like Catalytic Mechanism. RNA 14 (8), 1663-1670. doi:10. 1261/rna. 970408

Katze, J. R., Basile, B., and McCloskey, J. A. (1982). Queuine, a Modified Base Incorporated Posttranscriptionally into Eukaryotic Transfer RNA: Wide Distribution in Nature. Science 216 (4541), 55-56. doi:10.1126/science.7063869

Kelly, D., Kotliar, M., Woo, V., Jagannathan, S., Whitt, J., Moncivaiz, J., et al. (2018). Microbiota-sensitive Epigenetic Signature Predicts Inflammation in Crohn's Disease. JCI Insight 3 (18). doi:10.1172/jci.insight.122104

Kim, Y.-I. (2005). Nutritional Epigenetics: Impact of Folate Deficiency on DNA Methylation and colon Cancer Susceptibility. J. Nutr. 135 (11), 2703-2709. doi:10.1093/jn/135.11.2703

Kirtland, G. M., Morris, T. D., Moore, P. H., O'Brian, J. J., Edmonds, C. G., McCloskey, J. A., et al. (1988). Novel Salvage of Queuine from Queuosine and Absence of Queuine Synthesis in Chlorella Pyrenoidosa and Chlamydomonas Reinhardtii. J. Bacteriol. 170 (12), 5633-5641. doi:10.1128/jb.170.12.5633-5641. 1988

Krautkramer, K. A., Kreznar, J. H., Romano, K. A., Vivas, E. I., Barrett-Wilt, G. A., Rabaglia, M. E., et al. (2016). Diet-Microbiota Interactions Mediate Global Epigenetic Programming in Multiple Host Tissues. Mol. Cel. 64 (5), 982-992. doi:10.1016/j.molcel.2016.10.025

Krautkramer, K. A., Rey, F. E., and Denu, J. M. (2017). Chemical Signaling between Gut Microbiota and Host Chromatin: What Is Your Gut Really Saying? J. Biol. Chem. 292 (21), 8582-8593. doi:10.1074/jbc.R116.761577

Krtenic, B., Drazic, A., Arnesen, T., and Reuter, N. (2020). Classification and Phylogeny for the Annotation of Novel Eukaryotic GNAT Acetyltransferases. Plos Comput. Biol. 16 (12), e1007988. doi:10.1371/journal.pcbi.1007988

La Rosa, S. L., Leth, M. L., Michalak, L., Hansen, M. E., Pudlo, N. A., Glowacki, R., et al. (2019). The Human Gut Firmicute Roseburia Intestinalis Is a Primary Degrader of Dietary $\beta$-mannans. Nat. Commun. 10 (1), 905. doi:10.1038/ s41467-019-08812-y

Lavi, T., Siman-Tov, R., and Ankri, S. (2008). EhMLBP Is an Essential Constituent of theEntamoeba Histolyticaepigenetic Machinery and a Potential Drug Target. Mol. Microbiol. 69 (1), 55-66. doi:10.1111/j.1365-2958.2008.06258.xMMI6258

Lee, K. K., and Workman, J. L. (2007). Histone Acetyltransferase Complexes: One Size Doesn't Fit All. Nat. Rev. Mol. Cel. Biol. 8 (4), 284-295. doi:10.1038/nrm2145

Leitsch, D. (2019). A Review on Metronidazole: an Old Warhorse in Antimicrobial Chemotherapy. Parasitology 146 (9), 1167-1178. doi:10.1017/S0031182017002025

Linford, A. S., Moreno, H., Good, K. R., Zhang, H., Singh, U., and Petri, W. A., Jr. (2009). Short Hairpin RNA-Mediated Knockdown of Protein Expression in Entamoeba Histolytica. BMC Microbiol. 9, 38. doi:10.1186/1471-2180-9-38

Lopez-Siles, M., Duncan, S. H., Garcia-Gil, L. J., and Martinez-Medina, M. (2017). Faecalibacterium Prausnitzii: from Microbiology to Diagnostics and Prognostics. ISME J. 11 (4), 841-852. doi:10.1038/ismej.2016.176

Lozano-Amado, D., Ávila-López, P. A., Hernández-Montes, G., Briseño-Díaz, P., Vargas, M., Lopez-Rubio, J.-J., et al. (2020). A Class I Histone Deacetylase Is Implicated in the Encystation of Entamoeba Invadens. Int. J. Parasitol. 50 (12), 1011-1022. doi:10.1016/j.ijpara.2020.05.014

Lozano-Amado, D., Herrera-Solorio, A. M., Valdés, J., Alemán-Lazarini, L., Almaraz-Barrera, M. d. J., Luna-Rivera, E., et al. (2016). Identification of Repressive and Active Epigenetic marks and Nuclear Bodies in Entamoeba Histolytica. Parasites Vectors 9, 19. doi:10.1186/s13071-016-1298-7

Lyko, F. (2018). The DNA Methyltransferase Family: a Versatile Toolkit for Epigenetic Regulation. Nat. Rev. Genet. 19 (2), 81-92. doi:10.1038/nrg.2017.80

Lynch, S. V., and Pedersen, O. (2016). The Human Intestinal Microbiome in Health and Disease. N. Engl. J. Med. 375 (24), 2369-2379. doi:10.1056/ NEJMra1600266

Mansour-Ghanaei, F., Dehbashi, N., Yazdanparast, K., and Shafaghi, A. (2003). Efficacy of saccharomyces Boulardii with Antibiotics in Acute Amoebiasis. Wig 9 (8), 1832-1833. doi:10.3748/wjg.v9.i8.1832 
Maslowski, K. M., and Mackay, C. R. (2011). Diet, Gut Microbiota and Immune Responses. Nat. Immunol. 12 (1), 5-9. doi:10.1038/ni0111-5

Masuda, I., Matsubara, R., Christian, T., Rojas, E. R., Yadavalli, S. S., Zhang, L., et al. (2019). tRNA Methylation Is a Global Determinant of Bacterial Multi-Drug Resistance. Cel Syst. 8 (5), 475. doi:10.1016/j.cels.2019.05.002

Matthiesen, J., Lender, C., Haferkorn, A., Fehling, H., Meyer, M., Matthies, T., et al. (2019). Trigger-induced RNAi Gene Silencing to Identify Pathogenicity Factors of Entamoeba Histolytica. FASEB j. 33 (2), 1658-1668. doi:10.1096/fj. 201801313R

Metodiev, M. D., Spåhr, H., Loguercio Polosa, P., Meharg, C., Becker, C., Altmueller, J., et al. (2014). NSUN4 Is a Dual Function Mitochondrial Protein Required for Both Methylation of $12 \mathrm{~S}$ rRNA and Coordination of Mitoribosomal Assembly. Plos Genet. 10 (2), e1004110. doi:10.1371/journal.pgen.1004110

Mirelman, D., Anbar, M., Nuchamowitz, Y., and Bracha, R. (2006). Epigenetic Silencing of Gene Expression in Entamoeba Histolytica. Arch. Med. Res. 37 (2), 226-233. doi:10.1016/j.arcmed.2005.09.003

Morf, L., Pearson, R. J., Wang, A. S., and Singh, U. (2013). Robust Gene Silencing Mediated by Antisense Small RNAs in the Pathogenic Protist Entamoeba Histolytica. Nucleic Acids Res. 41 (20), 9424-9437. doi:10.1093/nar/gkt717

Motorin, Y., and Helm, M. (2010). tRNA Stabilization by Modified Nucleotides. Biochemistry 49 (24), 4934-4944. doi:10.1021/bi100408z

Nagaraja, S., and Ankri, S. (2019). Target Identification and Intervention Strategies against Amebiasis. Drug Resist. Updates 44, 1-14. doi:10.1016/j.drup.2019. 04.003

Nagaraja, S., Cai, M. W., Sun, J., Varet, H., Sarid, L., Trebicz-Geffen, M., et al. (2021). Queuine Is a Nutritional Regulator of Entamoeba Histolytica Response to Oxidative Stress and a Virulence Attenuator. mBio 12 (2). doi:10.1128/mBio.03549-20

Nakada-Tsukui, K., and Nozaki, T. (2016). Immune Response of Amebiasis and Immune Evasion by Entamoeba Histolytica. Front. Immunol. 7, 175. doi:10. 3389/fimmu.2016.00175

Neis, E., Dejong, C., and Rensen, S. (2015). The Role of Microbial Amino Acid Metabolism in Host Metabolism. Nutrients 7 (4), 2930-2946. doi:10.3390/ nu7042930

Netzer, N., Goodenbour, J. M., David, A., Dittmar, K. A., Jones, R. B., Schneider, J. R., et al. (2009). Innate Immune and Chemically Triggered Oxidative Stress Modifies Translational Fidelity. Nature 462 (7272), 522-526. doi:10.1038/ nature 08576

Ngobeni, R., Samie, A., Moonah, S., Watanabe, K., Petri, W. A., Jr., and Gilchrist, C. (2017). Entamoeba Species in South Africa: Correlations with the Host Microbiome, Parasite Burdens, and First Description of Entamoeba Bangladeshi outside of Asia. J. Infect. Dis. 216 (12), 1592-1600. doi:10.1093/ infdis/jix 535

Ott, G., Kersten, H., and Nishimura, S. (1982). Dictyostelium discoideum: a Useful Model System to Evaluate the Function of Queuine and of the Q-Family of tRNAs. FEBS Lett. 146 (2), 311-314. doi:10.1016/00145793(82)80941-1

Padilla-Vaca, F., Ankri, S., Bracha, R., Koole, L. A., and Mirelman, D. (1999). Down Regulation of Entamoeba Histolytica Virulence by Monoxenic Cultivation with Escherichia coli O55 Is Related to a Decrease in Expression of the Light (35kilodalton) Subunit of the Gal/GalNAc Lectin. Infect. Immun. 67 (5), 2096-2102. doi:10.1128/iai.67.5.2096-2102.1999

Park, S.-Y., and Kim, J.-S. (2020). A Short Guide to Histone Deacetylases Including Recent Progress on Class II Enzymes. Exp. Mol. Med. 52 (2), 204-212. doi:10. 1038/s12276-020-0382-4

Paturi, S., and Deshmukh, M. V. (2021). A Glimpse of "Dicer Biology" through the Structural and Functional Perspective. Front. Mol. Biosci. 8, 643657. doi:10. 3389/fmolb.2021.643657

Phalke, S., Nickel, O., Walluscheck, D., Hortig, F., Onorati, M. C., and Reuter, G. (2009). Retrotransposon Silencing and Telomere Integrity in Somatic Cells of Drosophila Depends on the Cytosine-5 Methyltransferase DNMT2. Nat. Genet. 41 (6), 696-702. doi:10.1038/ng.360ng.360

Poirier, L. A., Wise, C. K., Delongchamp, R. R., and Sinha, R. (2001). Blood Determinations of S-Adenosylmethionine, S-Adenosylhomocysteine, and Homocysteine: Correlations with Diet. Cancer Epidemiol. Biomarkers Prev. 10 (6), 649-655.

Pompei, A., Cordisco, L., Amaretti, A., Zanoni, S., Matteuzzi, D., and Rossi, M. (2007). Folate Production by Bifidobacteria as a Potential Probiotic Property. Appl. Environ. Microbiol. 73 (1), 179-185. doi:10.1128/AEM.01763-06
Pompey, J. M., Foda, B., and Singh, U. (2015). A Single RNaseIII Domain Protein from Entamoeba Histolytica Has dsRNA Cleavage Activity and Can Help Mediate RNAi Gene Silencing in a Heterologous System. PLoS One 10 (7), e0133740. doi:10.1371/journal.pone. 0133740

Prentis, P. J., Wilson, J. R. U., Dormontt, E. E., Richardson, D. M., and Lowe, A. J. (2008). Adaptive Evolution in Invasive Species. Trends Plant Sci. 13 (6), 288-294. doi:10.1016/j.tplants.2008.03.004

Qin, Y., and Wade, P. A. (2018). Crosstalk between the Microbiome and Epigenome: Messages from Bugs. J. Biochem. 163 (2), 105-112. doi:10.1093/ $\mathrm{jb} / \mathrm{mvx} 080$

Quach, J., St-Pierre, J., and Chadee, K. (2014). The Future for Vaccine Development againstEntamoeba Histolytica. Hum. Vaccin. Immunother. 10 (6), 1514-1521. doi:10.4161/hv.27796

Ramakrishnan, G., Gilchrist, C. A., Musa, H., Torok, M. S., Grant, P. A., Mann, B. J., et al. (2004). Histone Acetyltransferases and Deacetylase in Entamoeba Histolytica. Mol. Biochem. Parasitol. 138 (2), 205-216. doi:10.1016/j.molbiopara.2004.09.002

Rani, R., Rizvi, M. A., Paul, J., Bhattacharya, S., Ahuja, V., and Murthy, R. S. (2006). Changes in Bacterial Profile during Amebiasis: Demonstration of Anaerobic Bacteria in ALA Pus Samples. Am. J. Trop. Med. Hyg. 75 (5), 880-885. doi:10. 4269/ajtmh.2006.75.880

Richard, P., Kozlowski, L., Guillorit, H., Garnier, P., McKnight, N. C., Danchin, A., et al. (2021). Queuine, a Bacterial-Derived Hypermodified Nucleobase, Shows protection in In Vitro Models of Neurodegeneration. PLoS One 16 (8), e0253216. doi:10.1371/journal.pone.0253216

Rigothier, M. C., Maccario, J., and Gayral, P. (1994). Inhibitory Activity of saccharomyces Yeasts on the Adhesion ofEntamoeba Histolytica Trophozoites to Human Erythrocytes In Vitro. Parasitol. Res. 80 (1), 10-15. doi:10.1007/bf00932617

Schaefer, M., and Lyko, F. (2010). Lack of Evidence for DNA Methylation of Invader4 Retroelements in Drosophila and Implications for Dnmt2Mediated Epigenetic Regulation. Nat. Genet. 42 (11), 920-921. doi:10. 1038/ng1110-920

Schaefer, M., Pollex, T., Hanna, K., Tuorto, F., Meusburger, M., Helm, M., et al. (2010). RNA Methylation by Dnmt2 Protects Transfer RNAs against StressInduced Cleavage. Genes Dev. 24 (15), 1590-1595. doi:10.1101/gad.58671024/ $15 / 1590$

Schimmel, P. (2018). The Emerging Complexity of the tRNA World: Mammalian tRNAs beyond Protein Synthesis. Nat. Rev. Mol. Cel Biol 19 (1), 45-58. doi:10. 1038/nrm.2017.77

Shaulov, Y., Shimokawa, C., Trebicz-Geffen, M., Nagaraja, S., Methling, K., Lalk, M., et al. (2018). Escherichia coli Mediated Resistance of Entamoeba Histolytica to Oxidative Stress Is Triggered by Oxaloacetate. Plos Pathog. 14 (10), e1007295. doi:10.1371/journal.ppat.1007295

Shinoda, S., Kitagawa, S., Nakagawa, S., Wei, F.-Y., Tomizawa, K., Araki, K., et al. (2019). Mammalian NSUN2 Introduces 5-methylcytidines into Mitochondrial tRNAs. Nucleic Acids Res. 47 (16), 8734-8745. doi:10.1093/nar/gkz575

Sievers, K., Welp, L., Urlaub, H., and Ficner, R. (2021). Structural and Functional Insights into Human tRNA Guanine Transgylcosylase. RNA Biol. 18, 382-396. doi:10.1080/15476286.2021.1950980

Solis, C. F., Santi-Rocca, J., Perdomo, D., Weber, C., and Guillén, N. (2009). Use of Bacterially Expressed dsRNA to Downregulate Entamoeba Histolytica Gene Expression. PLoS One 4 (12), e8424. doi:10.1371/journal.pone.0008424

Stengl, B., Meyer, E. A., Heine, A., Brenk, R., Diederich, F., and Klebe, G. (2007). Crystal Structures of tRNA-Guanine Transglycosylase (TGT) in Complex with Novel and Potent Inhibitors Unravel Pronounced Induced-Fit Adaptations and Suggest Dimer Formation upon Substrate Binding. J. Mol. Biol. 370 (3), 492-511. doi:10.1016/j.jmb.2007.04.008

Tan, J., McKenzie, C., Potamitis, M., Thorburn, A. N., Mackay, C. R., and Macia, L. (2014). The Role of Short-Chain Fatty Acids in Health and Disease. Adv. Immunol. 121, 91-119. doi:10.1016/B978-0-12-800100-4.00003-9

Thomas, T., and Voss, A. K. (2007). The Diverse Biological Roles of MYST Histone Acetyltransferase Family Proteins. Cell Cycle 6 (6), 696-704. doi:10.4161/cc.6.6. 4013

Thompson, D. M., and Parker, R. (2009). Stressing Out over tRNA Cleavage. Cell 138 (2), 215-219. doi:10.1016/j.cell.2009.07.001S0092-8674(09)00795-8

Tovy, A., and Ankri, S. (2010). Epigenetics in the Unicellular Parasite Entamoeba Histolytica. Future Microbiol. 5 (12), 1875-1884. doi:10.2217/fmb.10.140

Tovy, A., Siman Tov, R., Gaentzsch, R., Helm, M., and Ankri, S. (2010). A New Nuclear Function of the Entamoeba Histolytica Glycolytic Enzyme Enolase: the 
Metabolic Regulation of Cytosine-5 Methyltransferase 2 (Dnmt2) Activity. Plos Pathog. 6 (2), e1000775. doi:10.1371/journal.ppat.1000775

Tuorto, F., Liebers, R., Musch, T., Schaefer, M., Hofmann, S., Kellner, S., et al. (2012). RNA Cytosine Methylation by Dnmt2 and NSun2 Promotes tRNA Stability and Protein Synthesis. Nat. Struct. Mol. Biol. 19 (9), 900-905. doi:10. 1038/nsmb.2357nsmb.2357

Turkeltaub, J. A., McCarty, T. R., 3rd, and Hotez, P. J. (2015). The Intestinal Protozoa. Curr. Opin. Gastroenterol. 31 (1), 38-44. doi:10.1097/MOG.0000000000000135

Van Haute, L., Dietmann, S., Kremer, L., Hussain, S., Pearce, S. F., Powell, C. A., et al. (2016). Deficient Methylation and Formylation of Mt-tRNAMet Wobble Cytosine in a Patient Carrying Mutations in NSUN3. Nat. Commun. 7, 12039. doi:10.1038/ncomms12039

Varet, H., Shaulov, Y., Sismeiro, O., Trebicz-Geffen, M., Legendre, R., Coppée, J.-Y., et al. (2018). Enteric Bacteria Boost Defences against Oxidative Stress in Entamoeba Histolytica. Sci. Rep. 8 (1), 9042. doi:10. 1038/s41598-018-27086-w

Verma, A. K., Verma, R., Ahuja, V., and Paul, J. (2012). Real-time Analysis of Gut flora in Entamoeba Histolytica Infected Patients of Northern India. BMC Microbiol. 12, 183. doi:10.1186/1471-2180-12-1831471-2180-12-183

Waldecker, M., Kautenburger, T., Daumann, H., Busch, C., and Schrenk, D. (2008). Inhibition of Histone-Deacetylase Activity by Short-Chain Fatty Acids and Some Polyphenol Metabolites Formed in the colon. J. Nutr. Biochem. 19 (9), 587-593. doi:10.1016/j.jnutbio.2007.08.002

Walden, T., Reyniers, J. P., Hiatt, V., and Farkas, W. R. (1982). Yeast Cells Cannot Incorporate Queuine into Their tRNA. Exp. Biol. Med. 170 (3), 328-332. doi:10. 3181/00379727-170-41438

Wassmann, C., Hellberg, A., Tannich, E., and Bruchhaus, I. (1999). Metronidazole Resistance in the Protozoan Parasite Entamoeba Histolytica Is Associated with Increased Expression of Iron-Containing Superoxide Dismutase and Peroxiredoxin and Decreased Expression of Ferredoxin 1 and Flavin Reductase. J. Biol. Chem. 274 (37), 26051-26056. doi:10.1074/jbc.274.37.26051

Watanabe, K., Gilchrist, C. A., Uddin, M. J., Burgess, S. L., Abhyankar, M. M., Moonah, S. N., et al. (2017). Microbiome-mediated Neutrophil Recruitment via CXCR2 and protection from Amebic Colitis. Plos Pathog. 13 (8), e1006513. doi:10.1371/journal.ppat.1006513PPATHOGENS-D-17-00667

Wesel, J., Shuman, J., Bastuzel, I., Dickerson, J., and Ingram-Smith, C. (2021). Encystation of Entamoeba Histolytica in Axenic Culture. Microorganisms 9 (4), 873. doi:10.3390/microorganisms 9040873

Yang, X., Yang, Y., Sun, B.-F., Chen, Y.-S., Xu, J.-W., Lai, W.-Y., et al. (2017). 5-methylcytosine Promotes mRNA export - NSUN2 as the
Methyltransferase and ALYREF as an m5C Reader. Cell Res. 27 (5), 606-625. doi:10.1038/cr.2017.55

Yoshida, M., Kijima, M., Akita, M., and Beppu, T. (1990). Potent and Specific Inhibition of Mammalian Histone Deacetylase Both In Vivo and In Vitro by Trichostatin A. J. Biol. Chem. 265 (28), 17174-17179. doi:10.1016/s0021-9258(17)44885-x

Yuan, Y., Zallot, R., Grove, T. L., Payan, D. J., Martin-Verstraete, I., Šepić, S., et al. (2019). Discovery of Novel Bacterial Queuine Salvage Enzymes and Pathways in Human Pathogens. Proc. Natl. Acad. Sci. USA 116 (38), 19126-19135. doi:10. 1073/pnas. 1909604116

Zallot, R., Brochier-Armanet, C., Gaston, K. W., Forouhar, F., Limbach, P. A., Hunt, J. F., et al. (2014). Plant, Animal, and Fungal Micronutrient Queuosine Is Salvaged by Members of the DUF2419 Protein Family. ACS Chem. Biol. 9 (8), 1812-1825. doi:10.1021/cb500278k

Zhang, H., Pompey, J. M., and Singh, U. (2011). RNA Interference in Entamoeba Histolytica: Implications for Parasite Biology and Gene Silencing. Future Microbiol. 6 (1), 103-117. doi:10.2217/fmb.10.154

Zhang, W., An, Y., Qin, X., Wu, X., Wang, X., Hou, H., et al. (2021). Gut Microbiota-Derived Metabolites in Colorectal Cancer: The Bad and the Challenges. Front. Oncol. 11, 739648. doi:10.3389/fonc.2021.739648

Zhou, H., Yuan, Y., Wang, H., Xiang, W., Li, S., Zheng, H., et al. (2021). Gut Microbiota: A Potential Target for Cancer Interventions. Cmar Vol. 13, 8281-8296. doi:10.2147/CMAR.S328249

Conflict of Interest: The authors declare that the research was conducted in the absence of any commercial or financial relationships that could be construed as a potential conflict of interest.

Publisher's Note: All claims expressed in this article are solely those of the authors and do not necessarily represent those of their affiliated organizations, or those of the publisher, the editors, and the reviewers. Any product that may be evaluated in this article, or claim that may be made by its manufacturer, is not guaranteed or endorsed by the publisher.

Copyright (C) 2022 Sarid and Ankri. This is an open-access article distributed under the terms of the Creative Commons Attribution License (CC BY). The use, distribution or reproduction in other forums is permitted, provided the original author(s) and the copyright owner(s) are credited and that the original publication in this journal is cited, in accordance with accepted academic practice. No use, distribution or reproduction is permitted which does not comply with these terms. 